\title{
MicroRNA-107 promotes apoptosis of acute myelocytic leukemia cells by targeting RAD51
}

\author{
Fengxia Huang ${ }^{1}$, Wei Tang ${ }^{2}$, Yan Lei $\mathrm{i}^{3,4,5}$
}

\author{
${ }^{1}$ Department of Medical Technology Clinical and Hematological Laboratory Office, \\ Xi'an Medical University, Xi'an, Shaanxi, China \\ ${ }^{2}$ Department of Radiology, Affiliated Hospital of North Sichuan Medical College, \\ Nanchong, Sichuan, China \\ ${ }^{3}$ Department of Laboratory Medicine, North Sichuan Medical College, Nanchong, \\ Sichuan, China \\ ${ }^{4}$ Department of Clinical Laboratory, Affiliated Hospital of North Sichuan Medical College, \\ Nanchong, Sichuan, China \\ ${ }^{5}$ Translational Medical Research Center, North Sichuan Medical College, Nanchong, \\ Sichuan, China
}

Submitted: 18 January 2018; Accepted: 11 June 2018

Online publication: 4 February 2020

Arch Med Sci 2021; 17 (4): 1044-1055

DOI: https://doi.org/10.5114/aoms.2020.92860

Copyright (c) 2020 Termedia \& Banach

\section{Abstract}

Introduction: This study aimed to investigate the role of microRNA (miRNA) that affects acute myelocytic leukemia (AML) and its potential molecular mechanism by constructing a miRNA-mRNA interaction network using bioinformatics methods.

Material and methods: MicroRNA expression data of AML were retrieved from Gene Expression Omnibus (GEO) and analyzed by microarray analysis. Expression levels of miR-107 and RAD51 mRNA were detected by quantitative real time polymerase chain reaction ( $q R T-P C R)$. Protein expression of RAD51, pro-apoptotic protein Bax, apoptosis related protein CytC and anti-apoptotic protein $\mathrm{Bcl}-2$ were determined by Western blot. The rate of cell apoptosis was detected by Annexin-V/PI. The predicted targeting relationship between miR-107 and the 3'UTR of RAD51 was first predicted by the online application TargetScan and then verified by dual-luciferase assay. Results: Acute myelocytic leukemia-associated genes $(n=197)$ and miRNAs $(n=1701)$ were retrieved from the database, the interaction network of miRNA-mRNA was constructed and the core position was occupied by RAD51. miR-107 exhibited a regulatory effect on RAD51 in which the MRNA and protein expression of RAD51 were both significantly inhibited by miR-107 mimics in vitro. Additionally, down-regulated expression of miR107 as well as up-regulated expression of RAD51 were detected not only in the plasma of AML patients compared to healthy volunteers, but also in AML cell lines compared to the normal bone marrow stromal cell line. Further study found that increased expression of miR-107 and the consequent down-regulation of RAD51 could aggravate the apoptosis of AML cells in vitro.

Conclusions: Our present results showed that the crucial role of RAD51 and miR-107 in the apoptosis of AML cells, i.e., miR-107 promotes the apoptosis of AML cells through down-regulating the expression of RAD51.

Key words: acute myelocytic leukemia, RAD51, MiR-107, apoptosis, interaction network.

\section{Introduction}

Acute myelocytic leukemia (AML) is a kind of myeloid malignancy that is often accompanied by the abnormal replication of bone marrow

\author{
Corresponding author: \\ Fengxia Huang \\ Department of Medical \\ Technology Clinical \\ and Hematological \\ Laboratory Office \\ Xi'an Medical University \\ No. 1 Xinwang Road \\ Weiyang District \\ 710021 Xi'an, China \\ E-mail: 252237256@qq.com
}


(BM) or peripheral blood (PB) cells [1]. Acute myelocytic leukemia is prevalent among children and adults with a higher proportion in adults. The incidence also increases with the growth of age, and the maximum age of $A M L$ patients even reaches 60 years old [2]. In the clinical treatment of $A M L$, it has been found that some AML patients in middle and old age achieved complete remission after treatment, while many more of these patients may face relatively high recurrence and a low survival rate [3]. As a blood-related tumor, AML is closely linked with hematopoiesis, a process mainly regulated by the effect of epigenetic modification [4, 5]. Methylation of DNA and histone are both common epigenetic modifications; interestingly, genes associated with DNA or histone methylation are usually mutated in AML $[6,7]$. For instance, HOX is often hypomethylated, whereas DNMT3A is often mutated in AML [8]. These dysfunctions in the processes of epigenetic modifications are among the possible causes of the development and recurrence of AML [9].

The previous studies about the marker genes in $A M L$ have reported that miRNA was one of the most commonly used biomarkers in adult AML patients [10]. MiRNA is a small non-coding RNA, which is prevalent in humans, animals, plants and some viruses and is highly conserved. Various developmental and physiological processes are adjusted by them, including cell division, immune response and cell apoptosis [11]. Through analyzing the expression of miRNA in tumor cells, it could be easily found that some miRNAs were abnormally expressed in a variety of cancer cells. Since miRNA was found, a large number of miRNAs were thought to be carcinogenic factors, while some miRNAs had the function of tumor suppressors. Studies about the function of miRNA in AML have gradually increased in recent decades. Researchers illustrated that miRNAs were crucial in the development of AML. For example, the absence of miR-145 and miR-146a could lead to long-term blood diseases in mice, and the introduction of these two miRNAs into AML cell lines could significantly induce cell death and slow the growth of cancer cells [12]. In addition, the expression of miR-29a, miR-224, miR-382 and miR-368 in $A M L$ patients was all different from that in healthy people, among which the first three miRNAs presented obviously higher expression in $A M L$ patients than in the healthy people $[13,14]$. These results suggested that miRNA played a critical role in the migration and growth of $A M L$ cells. However, scarce research on the mechanism of miRNA in AML was found, so there is an emergent need to study targets of miRNA as well as its mechanism.

The human RAD51 gene is located on chromosome $15 q 15.1$. Its protein product was found to be crucial for repairing dsDNA breaks and maintaining genomic diversity and stability, through the activity of eukaryotic homologous recombinase [15]. Studies suggested that slight changes in the RAD51 gene could cause DNA instability and lead to malignancies [16]. Cells lacking RAD51 are characterized by accumulation of chromosomal breaks before cell death. RAD51 gene knock-out in mice is embryonically lethal [17].

In this study, a lot of miRNAs that might be associated with AML were identified through analyzing the chip data in the GEO database. Then a key miRNA, miR-107, and its possible target gene RAD51 were determined using a miRNA-mRNA interaction network and dual luciferase reporter assay. Subsequent experiments proved that the decreasing expression of RAD51 induced by the up-regulated miR-107 can promote apoptosis in $\mathrm{AML}$ cell lines. In summary, the potential mechanisms of AML and the role of miRNA in the development of AML have been investigated.

\section{Material and methods}

\section{Acute myelocytic leukemia-related genes retrieval and miRNA prediction}

Acute myelocytic leukemia-related genes were retrieved and obtained from the Online Mendelian Inheritance in Man (OMIM) database, Catalogue of Somatic Mutations in Cancer (COSMIC), Genetic Association Database (GAD) and literature in PubMed. The target genes of miRNA were predicted by DIANA, miRanda, mirBridge, PicTar2 and TargetScan. Then, AML-related genes and miRNAs were selected to construct an interaction network of miRNA and mRNA.

\section{Analysis of acute myelocytic leukemia- related miRNA expression data}

One GSE was included for analysis. There were 65 cases in the GSE49665 database, which included the miRNA expression data from $52 \mathrm{AML}$ patients and 13 healthy volunteers. Among them, 5 cases of PB, 5 cases of BM and 3 cases of CD34+ cells were used as healthy samples for miRNA microarray analysis. The differentially expressed miRNAs were screened with a screening condition that fold change $>2$ and $p<0.05$.

\section{Clinical sample collection}

Clinical sample collection was authorized by the ethic committee of the Affiliated Hospital of North Sichuan Medical College (Nanchong City, Sichuan, China); all of the participants provided a signed informed consent. Peripheral blood samples from diagnostic AML patients $(n=20)$ and healthy volunteers $(n=20)$ were collected 
Table I. qRT-PCR primer aequences

\begin{tabular}{|lc|}
\hline Primer & \multicolumn{1}{c|}{ Sequence $\left(5^{\prime}\right.$ - $\mathbf{3}^{\prime}$ ) } \\
\hline RAD51: & \\
\hline Forward primer & AACAGAAGACGGCAACTCGT \\
\hline Reverse primer & GGCAACAGCCTCCACAGTAT \\
\hline miR-107: & \\
\hline Forward primer & CGAGAGCTACACGTTCACGG \\
\hline Reverse primer & GTGTCGAGGGAAAAATAGGCTG \\
\hline GAPDH: \\
\hline Forward primer & AGCCACATCGCTCAGACAC \\
\hline Reverse primer & GCCCAATACGACCAAATCC \\
\hline U6: & \\
\hline Forward primer & ATTGGAACGATACAGAGAAGATT \\
\hline Reverse primer & GGAACGCTTCACGAATTTG \\
\hline
\end{tabular}

at the hematology department of the Affiliated Hospital of North Sichuan Medical College. Briefly, a total of 2-3 $\mathrm{ml}$ of venous blood was collected after overnight fasting (more than $8 \mathrm{~h}$ ), heparin sodium was added for anticoagulation, the blood samples were centrifuged at $4^{\circ} \mathrm{C}$ for $10 \mathrm{~min}(2000 \mathrm{r} /$ $\mathrm{min})$, the supernatant component were collected (i.e., plasma). Total RNAs were extracted by plasma RNA Extraction Kit (Qiagen, Hilden, Germany) based on the manufacturer's instructions.

\section{Cell culture}

Acute myelocytic leukemia cell lines HEL, NOMO-1, TF-1, THP-1 and normal human BMSC HS-5 were obtained from BeNa culture Collection (Beijing, China). The HEL cell line was conventionally cultured in RPMI-1640 medium containing 10\% fetal bovine serum (FBS), $2.5 \mathrm{~g} /$ glucose, $1.5 \mathrm{~g} / \mathrm{l} \mathrm{NaHCO}_{3}$ and $0.11 \mathrm{~g} / \mathrm{l}$ sodium pyruvate; NOMO-1, TF-1 and THP-1 cell lines were cultured in RPMI-1640 medium only containing 10\% FBS. The HS-5 cell line was cultured in high glucose Dulbecco's Modified Eagle Medium (DMEM) with the supplementation of $10 \%$ FBS, $4 \mathrm{mM}$ L-glutamine and sodium pyruvate. Cells were incubated at $37^{\circ} \mathrm{C}$ with $5 \% \mathrm{CO}_{2}$ and saturated humidity.

\section{Cell transfection}

All plasmids including pcDNA3.1-RAD51, pcDNA3.1 plasmid vectors, RAD51 siRNA and siRNA negative control, miR-107 mimics/negative control mimic and miR-107 inhibitor/negative control inhibitor were bought from GenePharma (Shanghai, China). The sequences of siRNA were as follows: siRNARAD51: 5'-GGTTAGAGCAGTGTGGCAT-3'; siRNA-NC: 5'-GGTAGCGGAGTGTGTACAT-3'. Cells were plated in 6-well plates at a density of $1 \times 10^{6}$ cells/well. When the concentration of cells arrived at $80-90 \%$ (about $24 \mathrm{~h}$ ), plasmids were transfected into cells by Lipofectamine 2000 (Invitrogen, Carlsbad, USA) following the manufacturer's instructions. After incubation for a certain period (24-72 h), cells were centrifuged and collected for further detection.

\section{RNA isolation and quantitative real-time PCR (qRT-PCR)}

Total RNA was collected from the cultured cells or AML patients' blood cells using TRIzol reagent (Invitrogen) complying with the manufacturer's instructions. Then a cDNA library was constructed using M-MLV Reverse Transcription Kit (Invitrogen) for the subsequent qRT-PCR reactions. QRTPCR was performed with a SYBR Premix Ex Taq kit (Takara, Japan) and detected by the ABI7500 Real-Time PCR system (Applied Biosystems, Foster City, CA, USA). GAPDH (for mRNAs) or U6 (for miRNAs) was used for normalization respectively. The quantification analysis was based on the $2^{-\Delta \Delta \mathrm{ct}}$ method. All primers we used are shown in Table I.

\section{Detection for cell apoptosis}

Cell apoptosis was detected by Annexin V-FITC/PI double staining. Cells were cultured to $5 \times 10^{5}-1 \times 10^{6} / \mathrm{ml}$, then $1 \mathrm{ml}$ of cell suspension was harvested and the medium was removed by low-speed centrifugation at $4^{\circ} \mathrm{C}(1000 \mathrm{r} / \mathrm{min})$. After washing with PBS buffer twice, cells were resuspended into $200 \mu \mathrm{l}$ of binding buffer containing $5 \mu \mathrm{l}$ of PI and $10 \mu \mathrm{l}$ of Annexin V-FITC and then incubated at room temperature for 15 min under a dark condition. Subsequently, after the addition of $300 \mu \mathrm{l}$ of binding buffer, cells were detected by flow cytometry at $488 \mathrm{~nm}$. After being excited, cells were determined with red or green fluorescence (FITC showed green fluorescence, and PI showed red fluorescence).

\section{Dual-luciferase reporter assay}

Possible mRNA targets of miRNA-107 were found by TargetScan (www.targetscan.org); the best target is the 3'UTR fragment of RDA51. The 3'UTR of the RAD51 fragment (wild-type or mutant) was sub-cloned into the pMIR-Report Luciferase vector (pmirGLO) at loci Xbal and Fsel (Youbio, Changsha, China). PmirGLO, pmirGLO-RAD51-wt1/wt2 or pmirGLO-RAD51-mut1/mut2 was co-transfected with miRNA-107 mimics or miR control into 293T cells using Lipofectamine 2000 (Invitrogen Inc., Carlsbad, CA, USA). Renilla luciferase vector PRL-SV50 (Promega, Madison, WI, USA) was co-transfected with above constructs and served as a spiked-in control. The cells were cultured and tested for about 2 days. Quantitative analysis of luciferase 


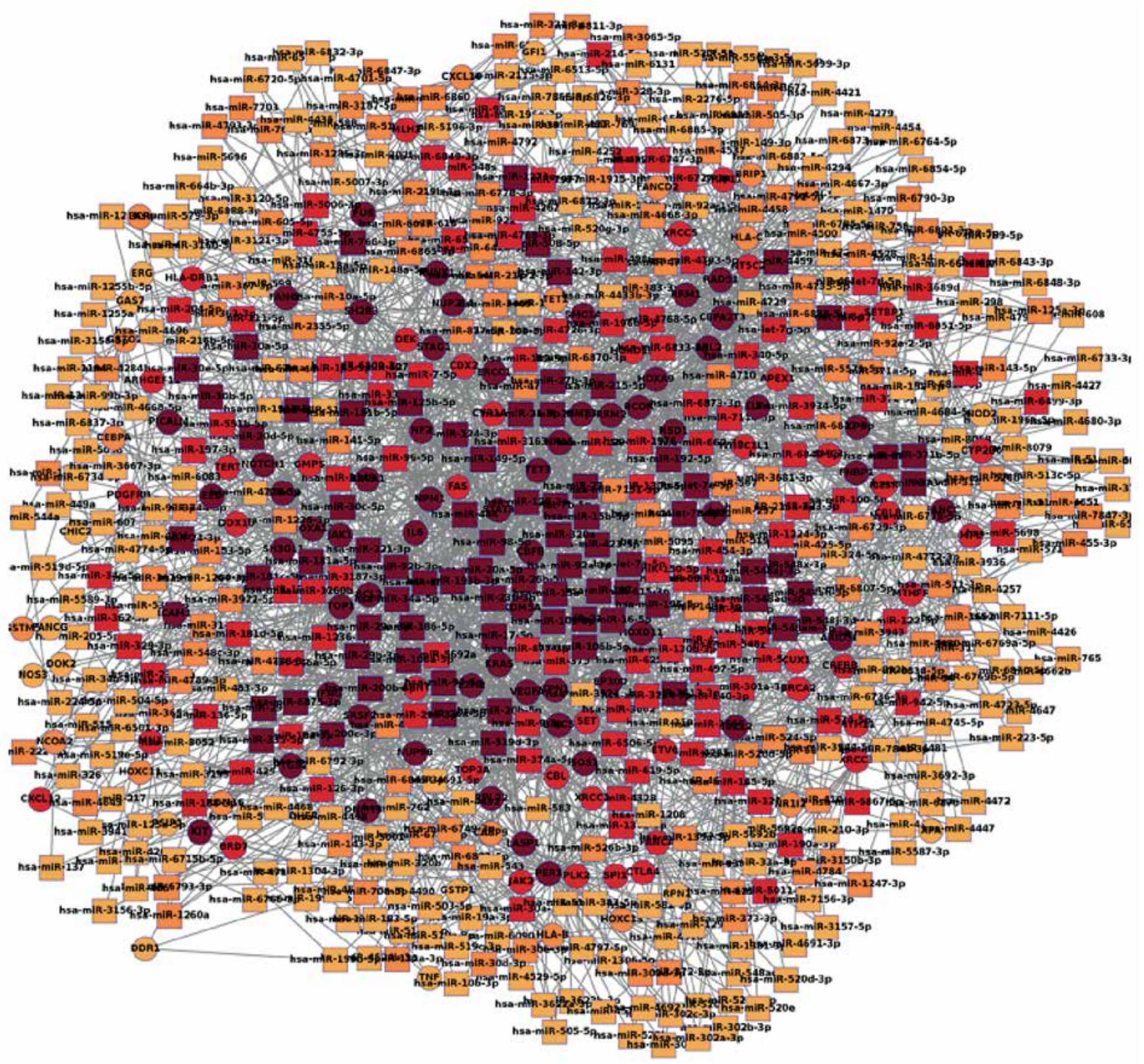

Figure 1. Acute myelocytic leukemia (AML)-related miRNA-mRNA interaction network. A total of $197 \mathrm{AML}$-related genes (non-repetitive) and miRNAs were obtained from OMIM, COSMIC, GAD and DisGeNET databases. Gene-miRNA interaction network was constructed by Dijkstra algorithm

activity was performed using a dual luciferase assay system (Promega). Relative luciferase activities were calculated as the ratios of firefly to Renilla luciferase activities.

\section{Western blot assay}

During the logarithmic growth phase, cells were collected and washed with PBS. Total protein was extracted with the addition of $1 \%$ SDS under icebath conditions and then quantified using a commercial protein assay kit (Thermo Fisher Scientific, Waltham, MA, USA). Subsequently, 50-100 $\mu \mathrm{g}$ of protein was separated by $12 \%$ SDS-PAGE gel and transferred to PVDF membranes. Membranes were blocked with TBS solution containing 5\% milk at normal temperature for $2 \mathrm{~h}$, and then were incubated with primary antibody against RAD51 (ab88572, 1 : 1000), Bax [E63] (ab32503, $1: 1000)$ and cytochrome C [7H8.2C12] (ab13575, $1: 2500)$ (Abcam, Cambridge, MA, USA) at $4^{\circ} \mathrm{C}$ overnight. The membranes were washed with TBST three times, the secondary antibody of HRP-labeled goat anti-rabbit IgG (ab6721, Abcam) antibody was then added to the membranes and incubated at room temperature for $1.5 \mathrm{~h}$. The antibody mixture was washed three times again with TBST buffer for 10 min each time and an ECL luminescent substrate was applied for visualization. The optical density values of the target strips were analyzed by Image J. $\beta$-actin was set as a control for normalization.

\section{Statistical analysis}

Statistical analysis was completed by GraphPad Prism 6.1 (Intuitive Software for Science, 
Table II. Acute myelocytic leukemia-related genes and core molecules in the network of miRNA (Top 20)

\begin{tabular}{|c|c|c|c|c|c|}
\hline miRNA & Kcore & Dgree & Gene & Kcore & Dgree \\
\hline hsa-miR-193b-3p & 6 & 60 & RAD51 & 6 & 224 \\
\hline hsa-miR-155-5p & 6 & 52 & HOXA13 & 6 & 214 \\
\hline hsa-miR-335-5p & 6 & 46 & VEGFA & 6 & 212 \\
\hline hsa-miR-26b-5p & 6 & 44 & TET3 & 6 & 208 \\
\hline hsa-miR-92a-3p & 6 & 44 & SRSF2 & 6 & 202 \\
\hline hsa-miR-17-5p & 6 & 36 & RRM2 & 6 & 200 \\
\hline hsa-miR-21-5p & 6 & 36 & PER1 & 6 & 192 \\
\hline hsa-let-7b-5p & 6 & 34 & ABL2 & 6 & 184 \\
\hline hsa-miR-98-5p & 6 & 34 & LPP & 6 & 180 \\
\hline hsa-let-7a-5p & 6 & 32 & ARID5B & 6 & 154 \\
\hline hsa-miR-124-3p & 6 & 32 & NSD1 & 6 & 154 \\
\hline hsa-miR-16-5p & 6 & 32 & SMC1A & 6 & 152 \\
\hline hsa-miR-192-5p & 6 & 32 & $\mathrm{BIRC5}$ & 6 & 148 \\
\hline hsa-miR-20a-5p & 6 & 30 & KDM5A & 6 & 148 \\
\hline hsa-miR-93-5p & 6 & 30 & NRAS & 6 & 146 \\
\hline hsa-miR-181a-5p & 6 & 28 & KRAS & 6 & 138 \\
\hline hsa-miR-218-5p & 6 & 28 & NF2 & 6 & 128 \\
\hline hsa-miR-34a-5p & 6 & 28 & HOXA9 & 6 & 124 \\
\hline hsa-let-7e-5p & 6 & 26 & STAT3 & 6 & 124 \\
\hline hsa-miR-484 & 6 & 26 & FNBP1 & 6 & 122 \\
\hline
\end{tabular}

San Diego, CA, USA). Mean \pm standard deviation (SD) was used to express the measurement data. Two tailed Student's $t$ test was used to compare the difference between two groups and one-way ANOVA analysis was used when there were more than two groups. Each experiment needed to be performed more than three times. $P<0.05$ was considered as the level of statistical significance.

\section{Results}

Acute myelocytic leukemia-related genes and miRNAs retrieval

A total of 197 AML-related genes (non-repetitive) were obtained from Online Mendelian Inheritance in Man (OMIM) database, Catalogue of Somatic Mutations in Cancer (COSMIC), Genetic Association Database (GAD) and Public Database (PDB). The names of these genes and the sources of these databases are shown in a supplementary document (Supplementary Table SI). MiRNAs interacting with AML were detected by DIANA, miRanda, mirBridge, PicTar2, TargetScan and miRNA-Target. Finally, 1701 miRNAs were successfully identified and had a targeted association with the above AML-related genes. The AML-related miRNA-mRNA interaction network was constructed (Figure 1) and the core genes or miRNAs were analyzed. According to the core degree from high to low, miRNAs and genes with a higher degree were selected as candidates for the subsequent analysis, including 76 core miRNAs and 60 core genes (Supplementary Table SII). The first 20 core miRNAs and genes are shown in Table II; it could be found that RAD51 is located at the center of the network of AML-related genes and miRNAs, indicating that RAD51 might play an important role in the development of AML.

\section{MicroRNA-107 was down-regulated} in acute myelocytic leukemia patients

MiRNA expression data of AML (GSE49665) were retrieved from the GEO database. Peripheral blood samples of AML patients were compared with healthy donor PB samples, BM samples and CD34+ cell samples, setting false discovery rates (FDRs) $<0.05$ for differential expression analysis. In contrast with healthy controls, there were 64 differential miRNAs in AML [18]. These 64 dif- 
A

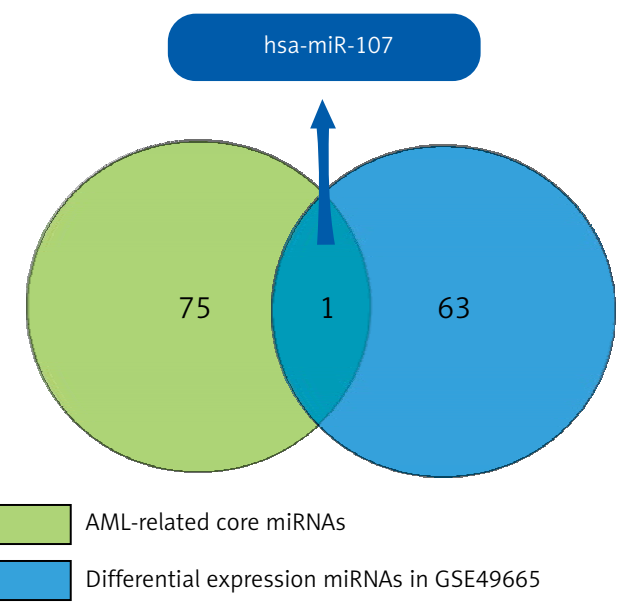

B

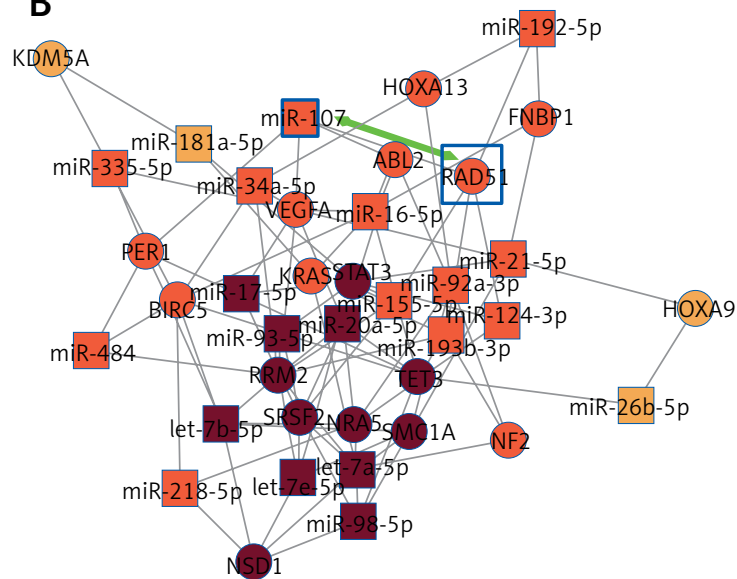

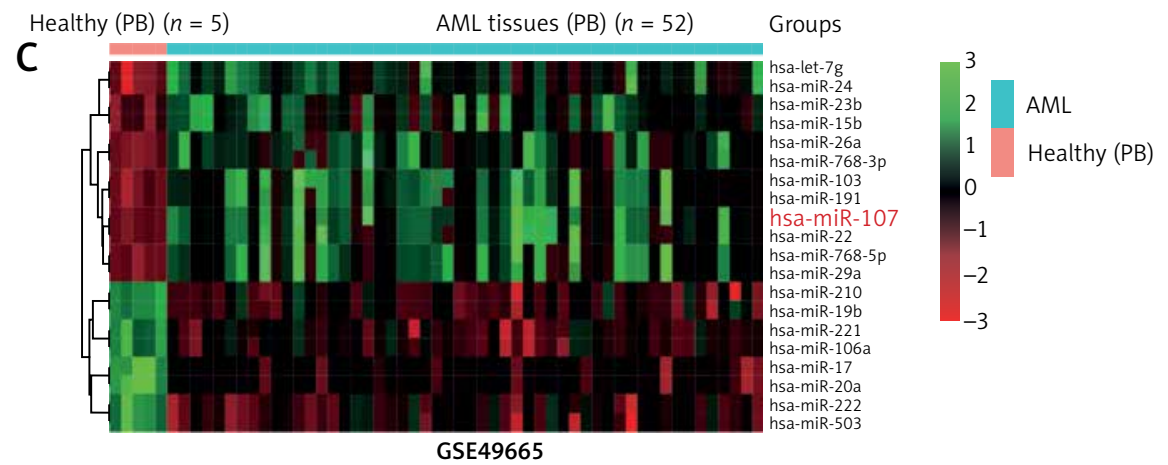

D

GSE49665

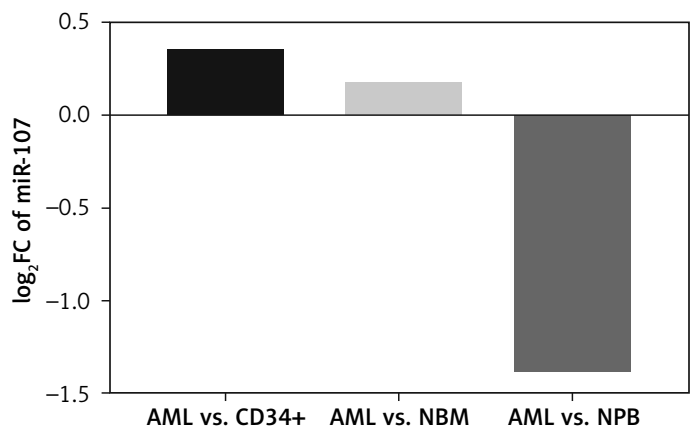

$\mathrm{E}$
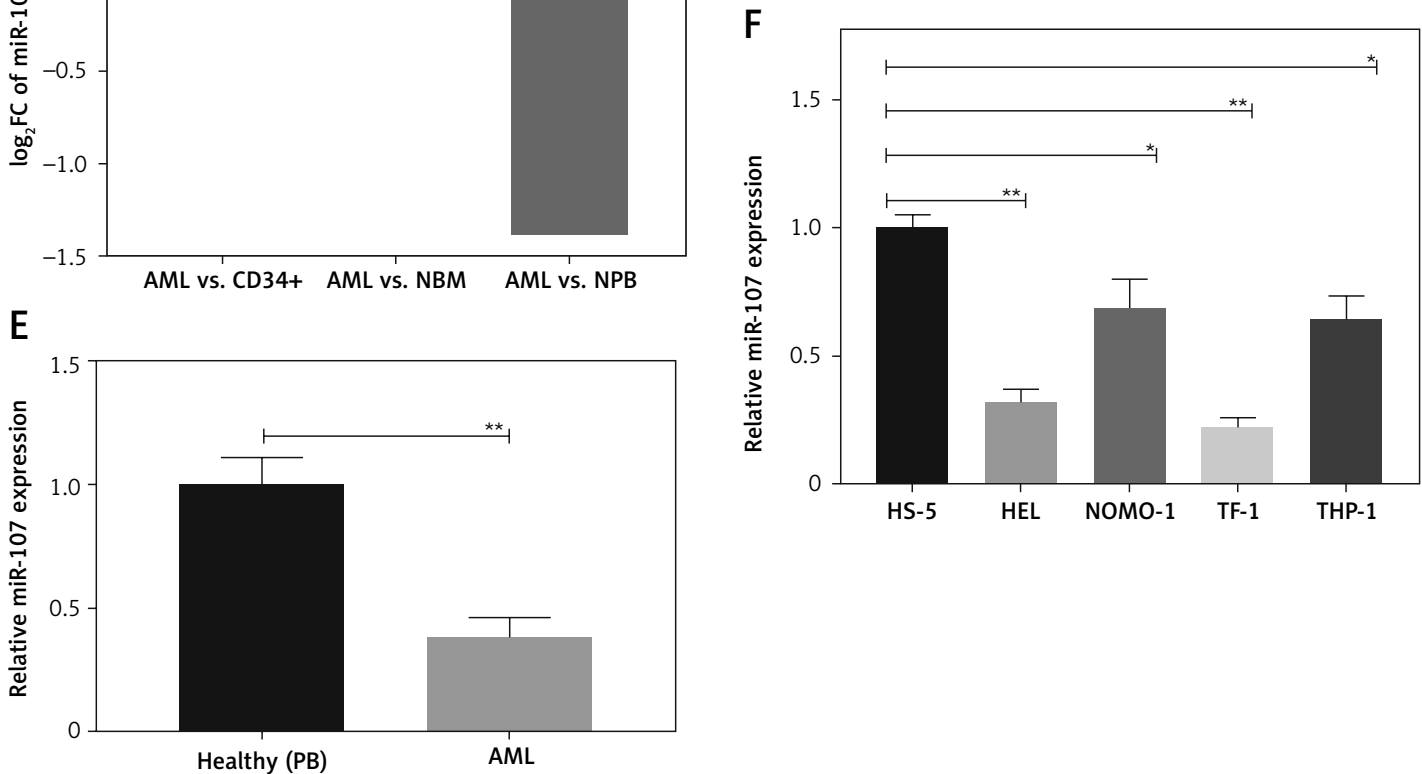

Figure 2. MicroRNA-107 was down-regulated in acute myelocytic leukemia (AML) samples. A - The collection of AML-related core miRNAs and differential expression miRNAs in GSE49665. B - MiR-107-related core gene sub-network. C - Expression profile of miRNAs in 52 AML peripheral blood samples compared with 5 peripheral blood (PB) healthy samples. Differentially expressed genes were labeled in heatmap and the threshold value was set as $p<0.05$, fold change value $>2$. D - Expression of miR-107 was downregulated in PB samples of AML patients compared with healthy PB samples, but upregulated in PB samples of AML patients compared with healthy CD34+ cell samples and BM samples. E- Expression of miR-107 from AML PB patients and healthy volunteers was verified by qRT-PCR. ${ }^{* \star} P<0.01$, compared with healthy (PB). $\mathrm{F}$ - The expression level of miR-107 was detected in AML PB cell lines HEL, NOMO-1, TF-1, THP-1 and bone marrow stromal cell line HS-5 by qRT-PCR. ${ }^{* *} P<0.01$, compared with HS- 5 
A

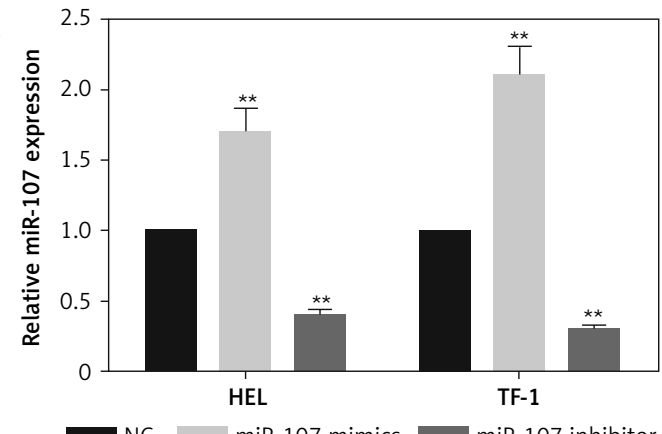

B

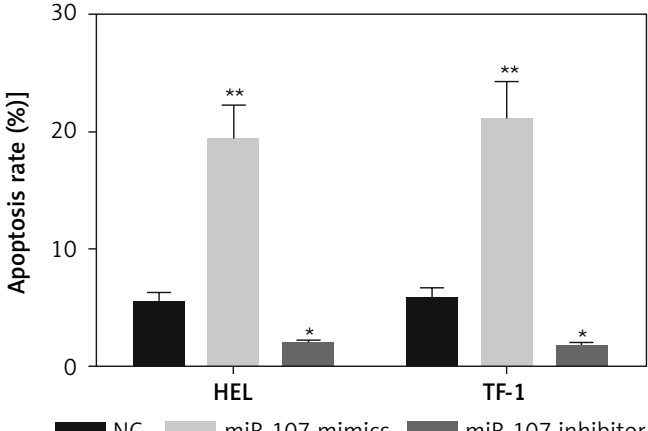

C

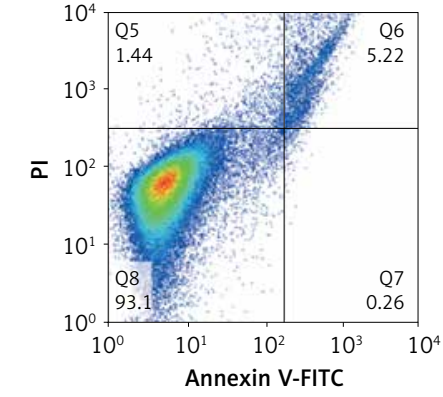

NC

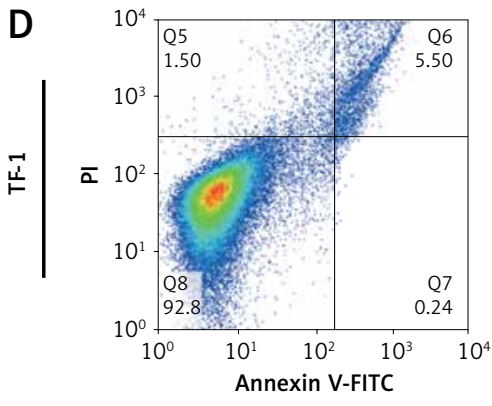

$\mathrm{NC}$

$E$
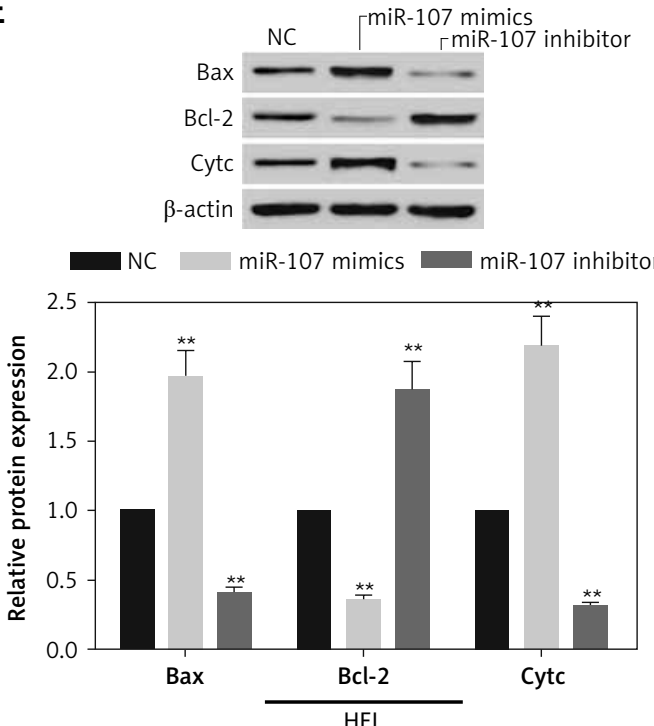

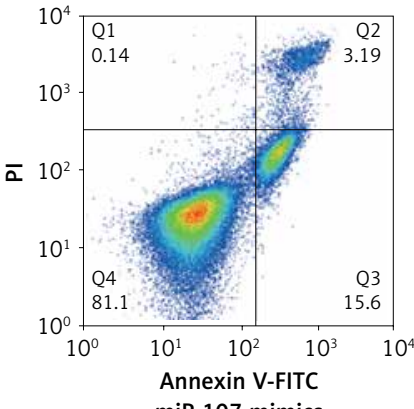

miR-107 mimics

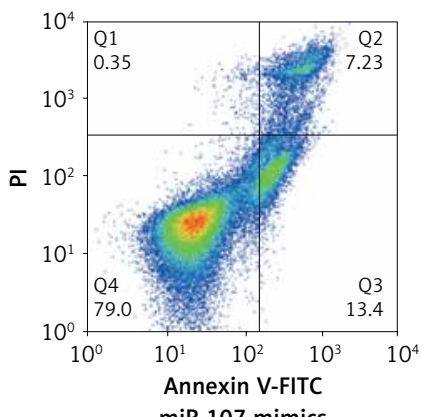

miR-107 mimics
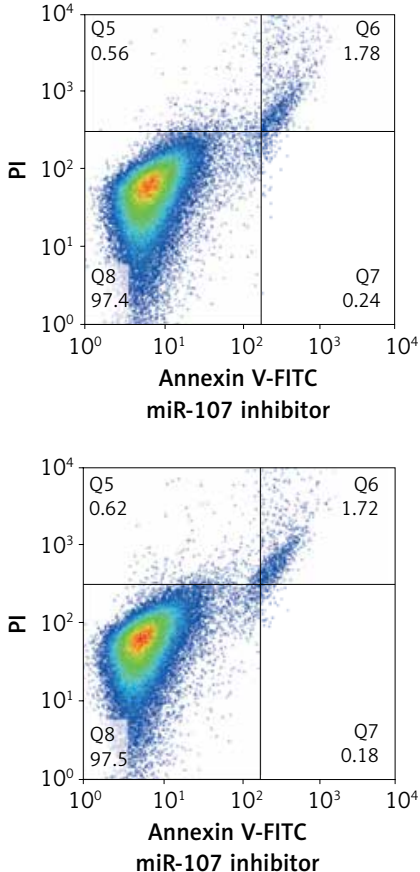

$\mathrm{F}$

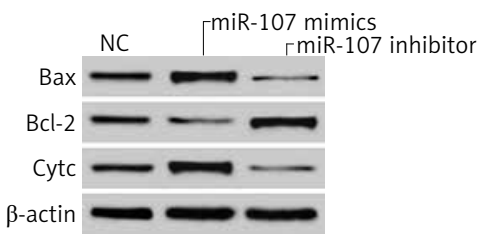

NC miR-107 mimics miR-107 inhibitor

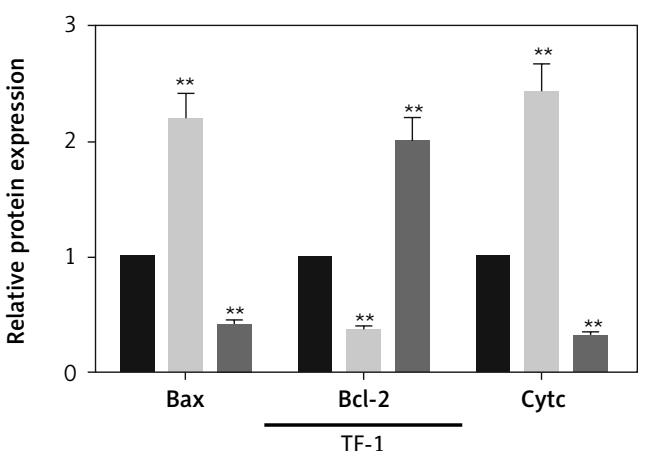

Figure 3. Effect of miR-107 on apoptosis of acute myelocytic leukemia (AML) cells. A-HEL and TF-1 cells were transfected with miR-107 mimics and miR-107 inhibitor and then divided into three groups including a miR-107 mimic group, miR-107 inhibitor group and negative control (NC) group. QRT-PCR detected miR-107 expression in HEL and TF-1 cells in different groups. B-D - Cell apoptosis was detected by flow cytometry, and the apoptosis rate was calculated in different transfected groups. E, F - expression levels of pro-apoptotic protein Bax, apoptosis related protein CytC and anti-apoptotic protein Bcl-2 in HEL (E) and TF-1 (F) cells was tested by Western blot. ${ }^{*} P<0.05$, ${ }^{* *} p<0.01$, compared with NC group 
ferential miRNAs were further co-analyzed with 76 core miRNAs from the prediction model of AML, and it was clear that there was a common miRNA of hsa-miR-107 (Figure 2 A). The targeted association between miR-107 and AML-related genes was examined by miRNA-Target. It was found that the AML-related gene RAD51 at the core position of the AML network had a targeted association with miR-107 (Figure $2 \mathrm{~B}$ ), indicating that the effect of miR-107 on AML might be achieved by affecting RAD51. By analyzing the GSE49665 chip based on the TU Graz miRNA array platform, 12 significantly down-regulated miRNAs including miR-107 were found in AML PB samples compared with $\mathrm{PB}$ healthy samples under the condition of FDRs less than 0.05 and $\log _{2}$ (fold change) $>1$ (Figure $2 \mathrm{C}$ ). But the expression level of miR-107 was higher in $P B$ of $A M L$ patients than healthy CD34+ cell and BM samples (Figure 2 D). These results showed that hsa-miR-107 was mainly differentially expressed in PB of AML patients. We further examined the miR-107 expression in AML patients and found that miR-107 was significantly downregulated in $\mathrm{AML}$ patients' samples compared with healthy patients' PB samples $(p<0.01$, Figure 2 E). Meanwhile, miR-107 expression level in different cells (AML cell lines HEL, NOMO-1, TF-1, THP-1 and normal human BMSC HS-5) was detected by qRT-PCR. It was demonstrated that the expression of miR-107 was significantly lowly expressed in cell lines HEL and TF-1 $(p<0.01)$ (Figure $2 \mathrm{~F}$ ). Thus, HEL and TF-1 were selected as research objects in the subsequent experiments.

\section{Effect of miR-107 on apoptosis of acute myelocytic leukemia cells}

MiR-107 mimics, miR-107 inhibitor and negative control (NC) were constructed and transfected into cell lines HEL and TF-1. When compared with the NC group, the miR-107 expression level was significantly increased in the miR-107 mimic group but decreased in the miR-107 inhibitor group $(p<0.01)$ (Figure $3 \mathrm{~A}$ ). The apoptosis rates of miR-107-transfected cells are shown in Figures 3 B-D; the apoptosis rate of HEL or TF-1 cells transfected in miR-107 mimics were significantly increased compared to the NC group ( $p<0.01$ ), but the apoptosis rate in the miR-107 inhibitor group was lower than that in the NC group $(p<0.05)$. In the meantime, the expression levels of protein Bax, CytC and $\mathrm{Bcl}-2$ in the transfected cells were analyzed by Western blot (Figures 3 E, F). Compared with the NC group, the expression level of CytC and Bax was significantly increase after up-regulating the expression of miR-107, while the expression level of $\mathrm{Bcl}-2$ was obviously decreased. These results showed that miR-107 could significantly promote the apoptosis of cell lines HEL and TF-1.

\section{RAD51 could directly target miR-107}

The results of online miRNA-target analysis (http://www.targetscan.org) showed that sites 9096 and 919-925 of the 3'UTR of RAD51 were both of the binding sites of hsa-miR-107 (Figure 4A). Subsequently, 3'UTR-RAD51-wt or 3'UTR-RAD51-mut and miR-107 mimics or miR-control were co-transfected into 293T cells. The activity of Firefly luciferase in the groups of 3'UTR-RAD51-wt1, 3'UTRRAD51-mut1 (90-96 sites) or 3'UTR-RAD51-mut2 (919-925 sites) co-transfected with miR-107 mimics were almost unchanged (Figures $4 \mathrm{~B}, \mathrm{C}$ ), but in the group of 3'UTR-RAD51-wt2 (919-925 sites) co-transfected with miR-107 mimics it was significantly decreased (Figure $4 \mathrm{C}$ ). The results suggested that miRNA-107 could act on the 919-925 sites of 3'UTR of RAD51, thereby down-regulating the expression of RAD51. QRT-PCR and Western blot showed that both RAD51 mRNA (Figure 4 D) and protein (Figure $4 \mathrm{E}$ ) were significantly up-regulated in $\mathrm{AML}$ patients' samples compared with PB healthy patients' samples. Linear correlation analysis showed that the expression of miR-107 has a negative relationship with $\operatorname{RAD51}\left(R^{2}=0.4555\right.$ and $\left.p<0.01\right)$ in AML patients (Figure $4 \mathrm{~F}$ ). Western blot showed that miR-107 mimics significantly inhibited the expression of RAD51 protein level in both HEL and TF-1 cell lines, whereas miR-107 inhibitor obviously promoted the expression of RAD51 protein (Figure $4 \mathrm{G)}$.

\section{Effect of RAD51 on acute myelocytic leukemia cell lines}

Si-RAD51 and pcDNA3.1-RAD51 were constructed and transfected into cell lines HEL and TF-1, respectively. The expression of RAD51 mRNA was markedly up-regulated in pcDNA-RAD51 group while it was down-regulated in the si-RAD51 group compared with the NC group in both HEL and TF- 1 cell lines $(p<0.01$, Figure 5 A). Western blot assay showed that the RAD51 protein expression level was highly expressed in the pcDNA-RAD51 group while it was lowly expressed in the si-RAD51 group compared with NC group in both HEL and TF-1 cell lines (Figure $5 \mathrm{~B}$ ). It was found that the apoptosis rate of $A M L$ in both $\mathrm{HEL}$ and TF-1 cell lines was significantly increased after silencing RAD51, whereas it was observably reduced after overexpression of RAD51 and this effect can be eliminated by the addition of miR-107 mimics (Figures 5 C, D). The expression of CytC, $\mathrm{Bax}$ and $\mathrm{Bcl}-2$ was detected by Western blot in HEL (Figure 5 E) and TF-1 (Figure 5 F) cell lines. Compared with the NC group, the expression level of CytC and Bax was obviously increased by suppressing the expression of RAD51 and was decreased by promoting the expression of RAD51, 
A

TagetScanHuman 7.1 miR-107 and position 90-96 of RAD51-3' UTR

RAD51-3' UTR-wt1 5'-GUUAAAAACCUUAAGUGCUGCAG-3'

hsa-miR-107 3'-ACUAUCGGGACAUGUUACGACGA-5'

RAD51-3' UTR-mut1 5'-GUUAAAAACCUUAAGACGACGAG-3'

TagetScanHuman 7.1 miR-107 and position 919-925 of RAD51-3' UTR

RAD51-3' UTR-wt2 5'-GAGUCGAGAUUGCACUGCUGCAU-3'

hsa-miR-107 3'-ACUAUCGGGACAUGUUACGACGA-5'

35a-miR-107 -ACUAUCGGGACAUGUUACGACGA-

RAD51-3' UTR-mut2 5'-GAGUCGAGAGAUUGCACACGACGAU-3'

C

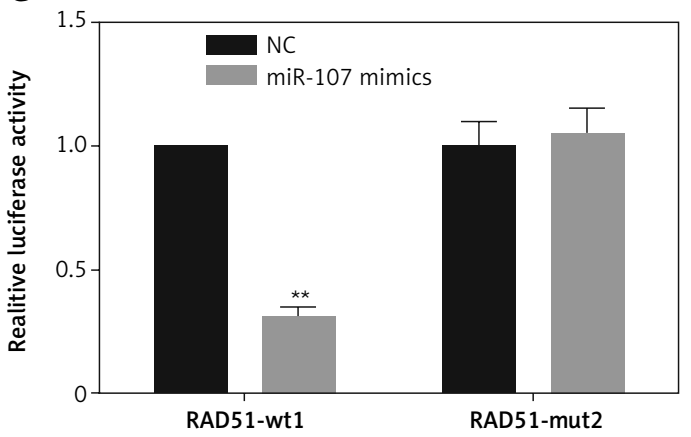

E
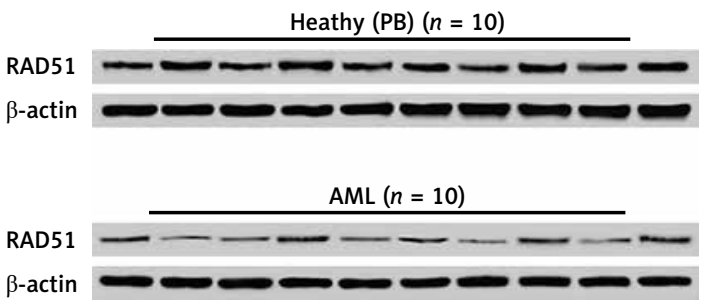

G

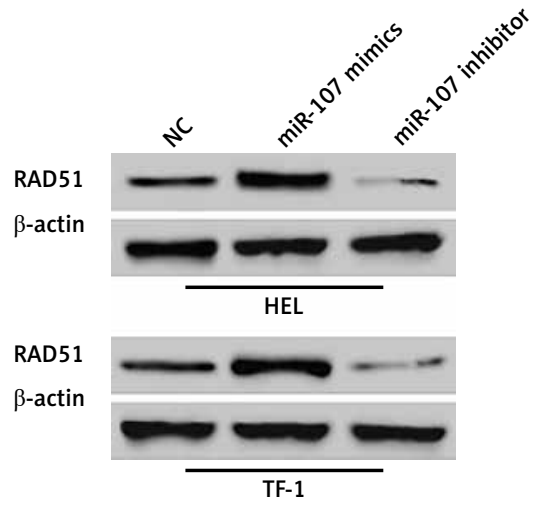

B

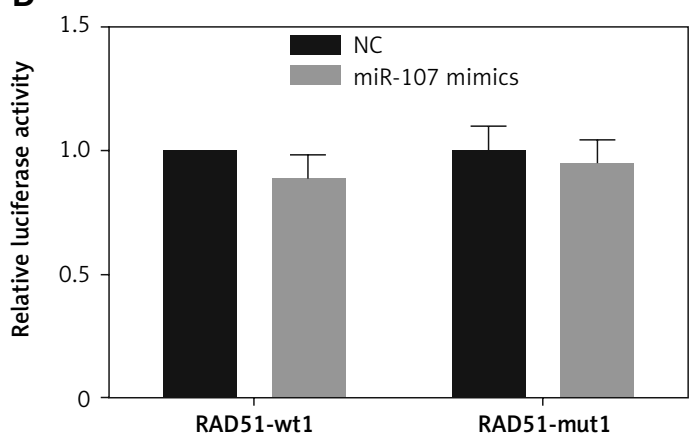

D

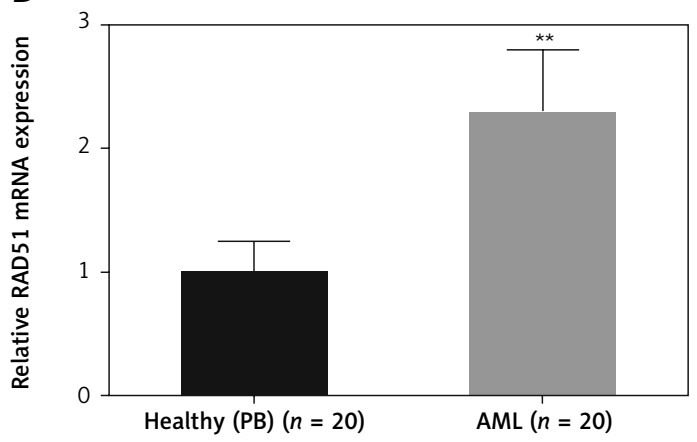

F
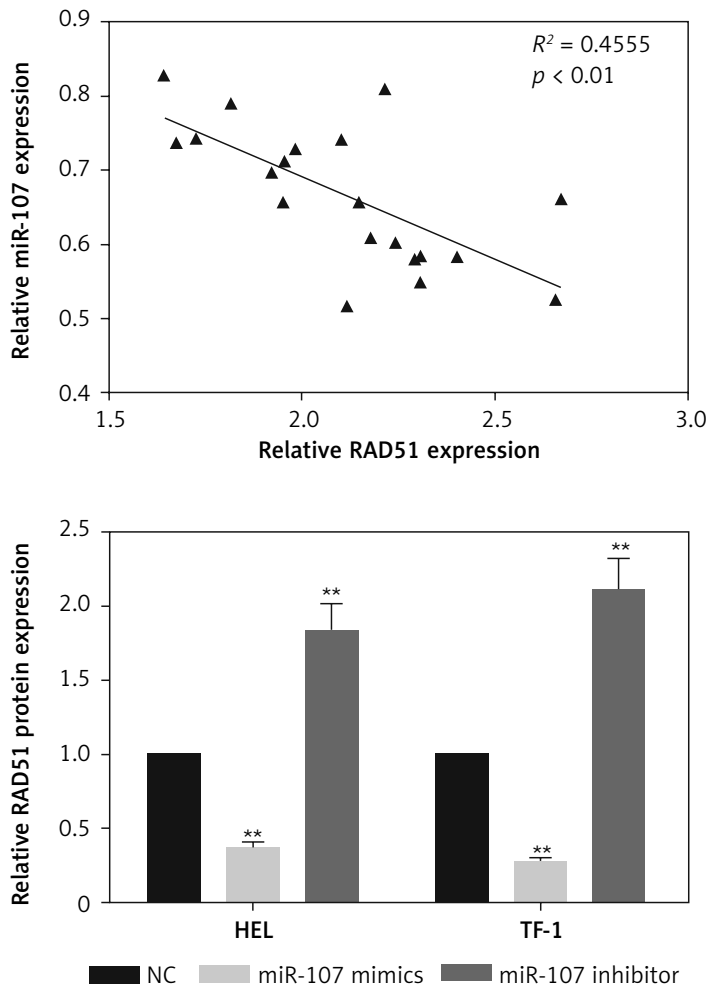

Figure 4. Effect of miR-107 on apoptosis of acute myelocytic leukemia (AML) cells. A - Prediction of binding sites of miR-107 and RAD51 by bioinformatics (TargetScan 7.1). B, C - Luciferase activity analysis, transfection of miR-107 mimics could significantly inhibit the RAD51 wild-type (wt1 and wt2) luciferase gene carrier fluorescence activity. ${ }^{* *} P<0.01$, compared with NC group. $\mathrm{D}$ - Expression of RAD51 mRNA was verified by qRT-PCR in AML PB patients compared with healthy PB volunteers. ${ }^{* *} P<0.01$, compared with healthy $(\mathrm{PB})$. $\mathrm{E}-$ Expression protein level of RAD51 in $10 \mathrm{AML}$ PB patients and healthy PB volunteers was detected by Western blot. F - Linear analysis revealed a negative correlation between RAD51 and miR-107 expression in AML PB patients' samples $(n=20)$. G - Protein expression level of RAD51 in HEL and TF-1 cells was tested by Western blot. ${ }^{* *} P<0.01$, compared with NC group 

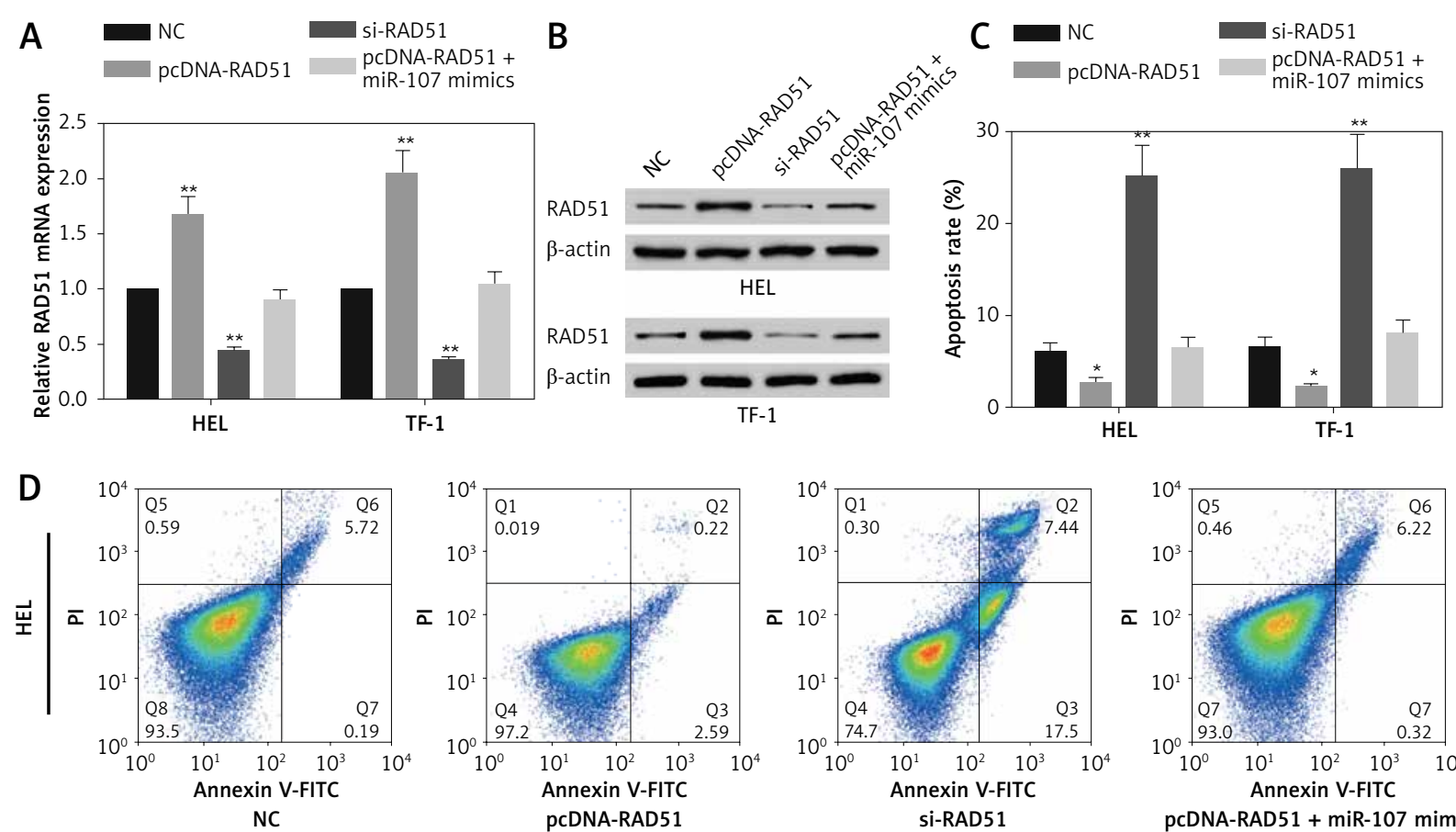

HEL

TF-1

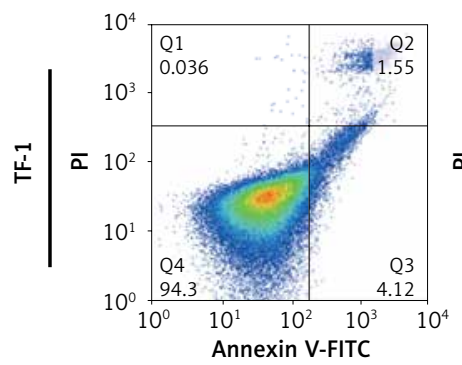

NC

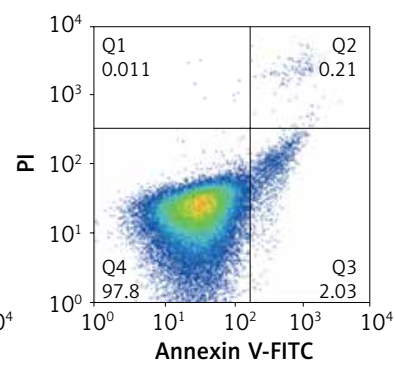

pCDNA-RAD51

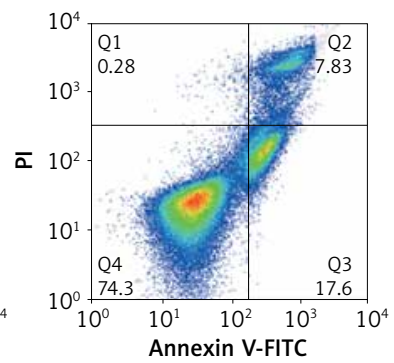

si-RAD51
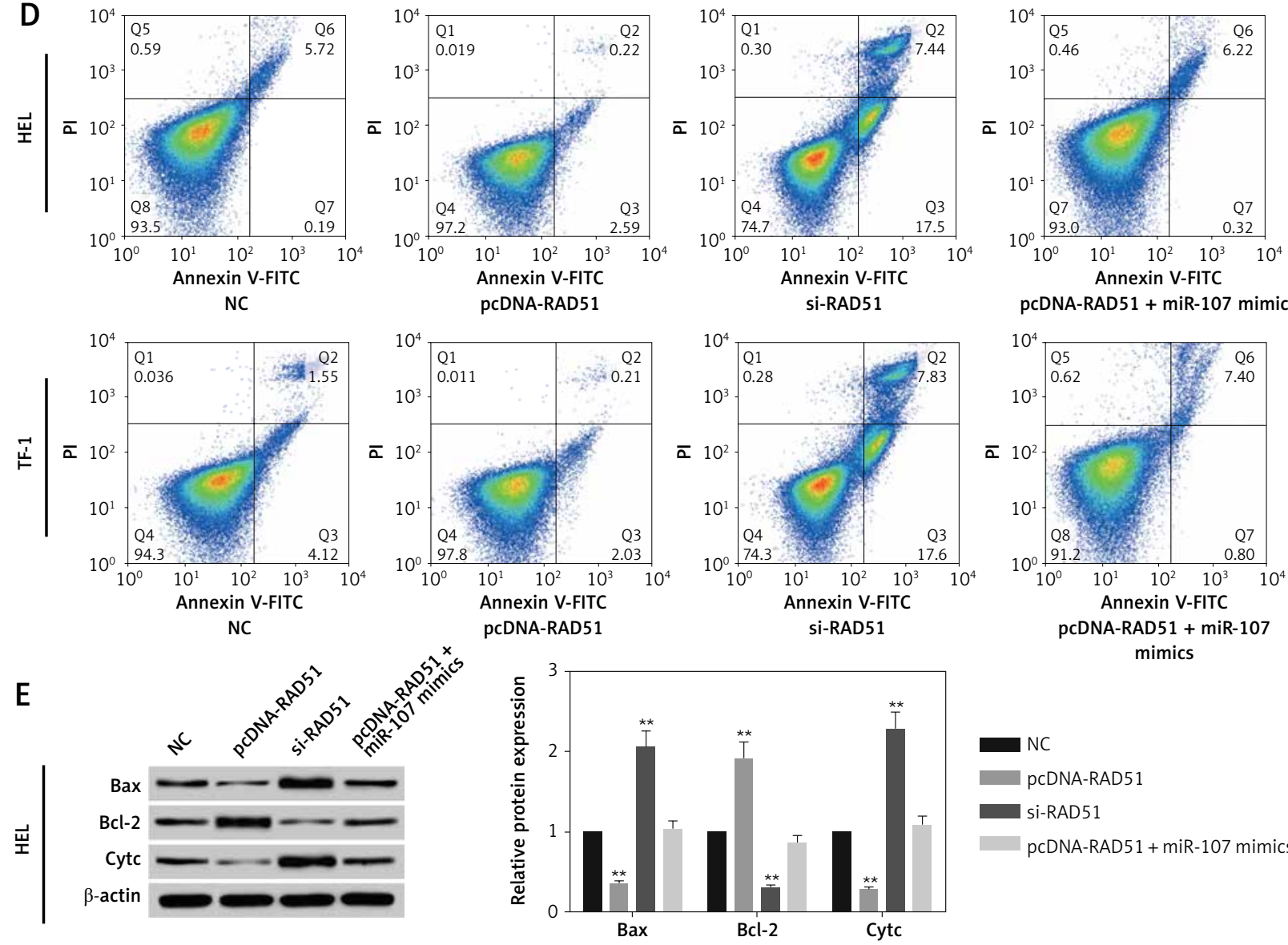

pCDNA-RAD51 + miR-107 mimics

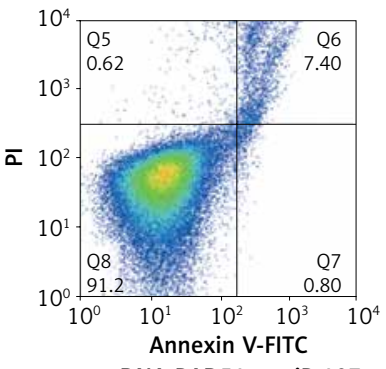

pCDNA-RAD51 + miR-107 mimics
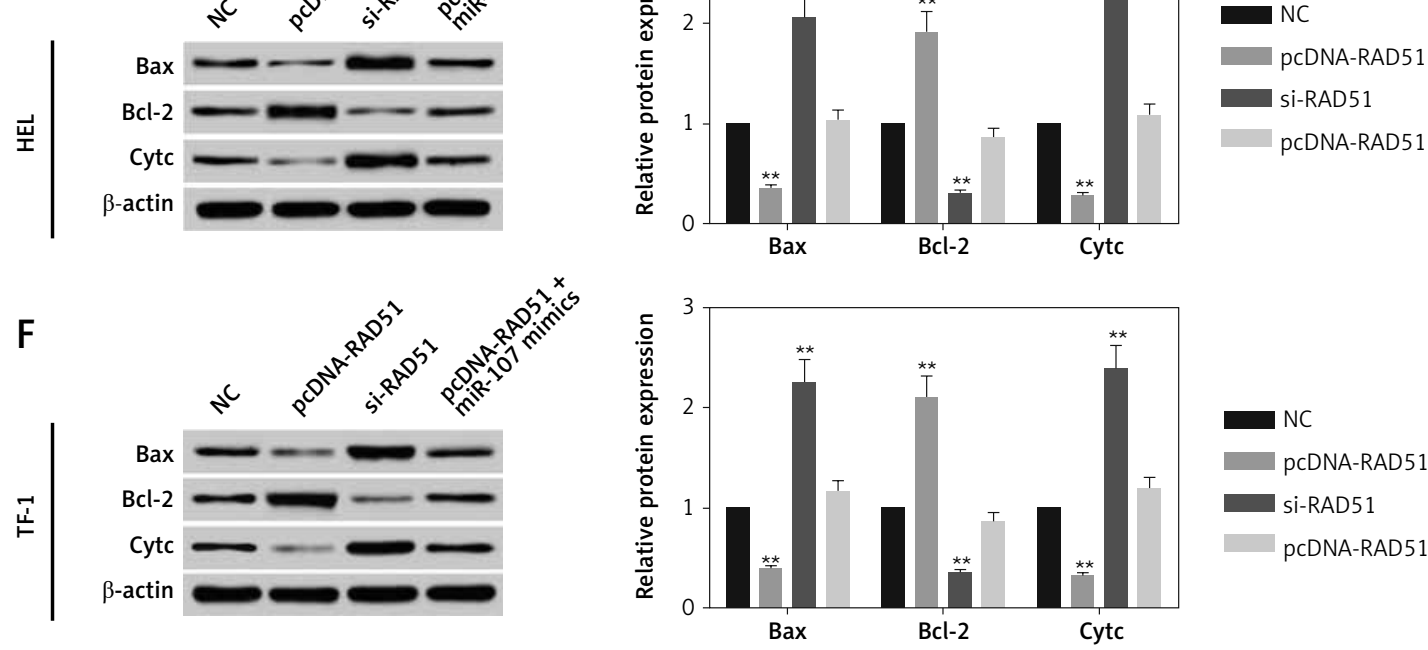

Si-RAD51

pcDNA-RAD51 + miR-107 mimics

Figure 5. Effect of RAD51 on apoptosis of acute myelocytic leukemia (AML) cells. A - HEL and TF-1 cells were transfected with pCDNA3.1-RAD51 and si-RAD51 and then divided into four groups including negative control (NC) group, pCDNA-RAD51 group, si-RAD51 group and pcDNA-RAD51 + miR-107 mimic group. QRT-PCR detected RAD51 mRNA expression in HEL and TF-1 cells in different transfected groups. B - Western blot detected RAD51 protein expression level in HEL and TF-1 cells in different transfected groups. C, D - Cell apoptosis was detected by flow cytometry, and the apoptosis rate was calculated in different transfected groups. E, F-Expression level of pro-apoptotic protein Bax, apoptosis related protein CytC and anti-apoptotic protein BCl-2 in HEL (E) and TF-1 (F) cells was tested by Western blot. ${ }^{*} P<0.05,{ }^{*} P<0.01$, compared with NC group 
while the expression level of $\mathrm{Bcl}-2$ was obviously reduced by suppressing the expression of RAD51 and was increased by promoting the expression of RAD51.

\section{Discussion}

Acute myelocytic leukemia is a complex heterogeneous disease that is often accompanied by large numbers of gene mutations [19]. Through the search and identification of AML-related genes, we can better understand the pathogenesis of AML, which could help us to find AML-associated biomarkers and therapeutic targets against this disease. Fortunately, at present, a growing number of public databases has facilitated the exploration of disease-related genes [20]. In this study, 197 non-repetitive AML-related genes were obtained from GAD, OMIM and COSMIC databases. Among them, genes such as VEGF, HOX and RAD51 showed a closer correlation with the development of AML. Furthermore, the RAD51 gene was found to be located at the central position of the constructed network of these AML-associated genes. RAD51 is a RecA-like DNA chain transferase protein that catalyzes the central reaction of homologous pairing and DNA chain exchange during homologous recombination [21]. Because of the key role of homologous recombination in the stability of cell genomes, most researchers agreed that there was a link between homologous recombination and cancer phenotype [22]. As a core protein in the process of homologous recombination, the expression of RAD51 was significantly increased in a variety of human tumor cells, which might cause drug resistance in tumor cells [23]. For example, in triple-negative breast cancer, the knockout of RAD51 could significantly inhibit the migration of tumor cells in vitro as well as the primary growth of tumors in vivo [24]; on the other hand, abated expression of RAD51 was also found in some other disease such as prostate cancer and colorectal cancers [25, 26]. Remarkably, it was found that inhibition of RAD51 resulted in apoptosis of AML cells [27]. These results suggested that RAD51 played a key role in the growth and apoptosis of tumors, but unfortunately the regulatory mechanism of RAD51 in the apoptosis of AML cells remains unclear.

To predict the possible key miRNAs in AML, the miRNAs possibly targeting AML-related genes were retrieved by TargetScan. The miRNA-mRNA interaction network was constructed, and core miRNAs with the highest likelihood $(n=76)$ were selected to compare with the reported AML-related miRNAs $(n=64)$. Finally, only miRNA-107 exists in both of our data and the reported data. MiR-107 is a miRNA located on chromosome 10. Previous studies found that miRNA was abnormally expressed in a variety of tumor cells [28-30]. Increased expression of miR-107 could significantly inhibit the growth, migration and invasion of various cancer cells, by targeting the different genes [31-33]. For example, miR107 directly inhibited the growth of gastric tumors by suppressing the expression of NF1 [31], and in non-small cell lung cancer, miR-107 could act on EGFR and thereby inhibit the proliferation and migration of tumor cells as well as promoting the apoptosis of tumor cells [34]. Here, database searches of the miRNA target showed that RAD51, the core network factor of AML, has binding sites of miR-107 and its expression is possibly regulated by the latter. Huang et al. found that miR-107 could directly combine with RAD51, and overexpression of miR-107 could significantly suppress the protein expression of RAD51 in various cancer cell lines [35]. Furthermore, previous studies have shown that in AML cells, multiple miRNAs including miR-107 were related to the apoptosis of AML tumor cells [36, 37]. In this study, we found that miR-107 could directly target RAD51 and inhibit its expression, but unlike with RAD51, the expression of apoptosis-associated genes was up-regulated. Subsequently, apoptosis of AML cells was triggered and then the growth of AML cells was significantly inhibited.

In conclusion, it was found that RAD51 was the direct target gene of miR-107 in AML by bioinformatics methods. Then, Western blot and cytological experiments demonstrated that in the $\mathrm{AML}$ cell lines, overexpression of miR-107 could significantly inhibit the expression of RAD51 and promote the death of AML cells. Subsequent experiments revealed that miR-107 was able to directly interact with the 3'UTR of RAD51. These results confirmed that miR-107 could promote the death of AML cells by inhibiting expression of RAD51.

In this study we firstly constructed an interaction network of AML-associated miRNA and mRNA by bioinformatics methods and revealed the potential roles of RAD51 and miR-107 in the apoptosis of AML cells. Further studies suggested that miR107 precisely promoted the apoptosis of AML cells through down-regulating the expression of RAD51.

\section{Acknowledgments}

This study was supported by the Third Instalment of Key Construction Subject Projects of Xi'an Medical University.

\section{Conflict of interest}

The authors declare no conflict of interest.

\footnotetext{
References

1. Meng CY, Noor PJ, Ismail A, Ahid MF, Zakaria Z. Cytogenetic profile of de novo acute myeloid leukemia patients in Malaysia. Int J Biomed Sci 2013; 9: 26-32.
} 
2. Shahjahani M, Khodadi E, Seghatoleslami M, et al. Rare cytogenetic abnormalities and alteration of microRNAs in acute myeloid leukemia and response to therapy. Oncol Rev 2015; 9: 261.

3. Estey EH. Acute myeloid leukemia: 2014 update on riskstratification and management. Am J Hematol 2014; 89: 1063-81.

4. Bock C, Beerman I, Lien WH, et al. DNA methylation dynamics during in vivo differentiation of blood and skin stem cells. Mol Cell 2012; 47: 633-47.

5. Ji H, Ehrlich LI, Seita J, et al. Comprehensive methylome map of lineage commitment from haematopoietic progenitors. Nature 2010; 467: 338-42.

6. Lokody I. Epigenetics: histone methyltransferase mutations promote leukaemia. Nat Rev Cancer 2014; 14: 214-5.

7. Cancer Genome Atlas Research Network, Ley TJ, Miller C, Ding $L$, et al. Genomic and epigenomic landscapes of adult de novo acute myeloid leukemia. N Engl J Med 2013; 368: 2059-74.

8. Qu Y, Lennartsson A, Gaidzik VI, et al. Differential methylation in CN-AML preferentially targets non-CGI regions and is dictated by DNMT3A mutational status and associated with predominant hypomethylation of $\mathrm{HOX}$ genes. Epigenetics 2014; 9: 1108-19.

9. Islam M, Mohamed Z, Assenov Y. Differential analysis of genetic, epigenetic, and cytogenetic abnormalities in AML. Int J Genomics 2017; 2017: 2913648.

10. Marcucci G, Mrozek K, Radmacher MD, Garzon R, Bloomfield $C D$. The prognostic and functional role of microRNAs in acute myeloid leukemia. Blood 2011; 117: 1121-9.

11. Bissels U, Bosio A, Wagner W. MicroRNAs are shaping the hematopoietic landscape. Haematologica 2012; 97: 160-7.

12. Starczynowski DT, Morin R, McPherson A, et al. Genomewide identification of human microRNAs located in leukemia-associated genomic alterations. Blood 2011; 117: 595-607.

13. Danen-van Oorschot AA, Kuipers JE, Arentsen-Peters S, et al. Differentially expressed miRNAs in cytogenetic and molecular subtypes of pediatric acute myeloid leukemia. Pediatr Blood Cancer 2012; 58: 715-21.

14. Li Z, Lu J, Sun M, et al. Distinct microRNA expression profiles in acute myeloid leukemia with common translocations. Proc Natl Acad Sci U S A 2008; 105: 15535-40.

15. He YZ, Hu X, Chi XS, et al. Association between RAD51 gene polymorphism $(-135 \mathrm{G} / \mathrm{C})$ and susceptibility of myelodysplastic syndrome and acute leukemia: evidence based on a meta-analysis. Tumour Biol 2014; 35: 615-21.

16. Thacker J. The RAD51 gene family, genetic instability and cancer. Cancer Lett 2005; 219: 125-35.

17. Liu L, Yang L, Mi Y, et al. RAD51 and XRCC3 polymorphisms: impact on the risk and treatment outcomes of de novo inv(16) or t(16;16)/CBFbeta-MYH11(+) acute myeloid leukemia. Leuk Res 2011; 35: 1020-6.

18. Rommer A, Steinleitner K, Hackl H, et al. Overexpression of primary microRNA 221/222 in acute myeloid leukemia. BMC Cancer 2013; 13: 364.

19. Saygin C, Carraway HE. Emerging therapies for acute myeloid leukemia. J Hematol Oncol 2017; 10: 93.

20. Névéol A, Wilbur WJ, Lu Z. Improving links between literature and biological data with text mining: a case study with GEO, PDB and MEDLINE. Database (Oxford) 2012; 2012: bas026.

21. Chen J, Morrical MD, Donigan KA, et al. Tumor-associated mutations in a conserved structural motif alter physical and biochemical properties of human RAD51 recombinase. Nucleic Acids Res 2015; 43: 1098-111.
22. Sung P, Klein H. Mechanism of homologous recombination: mediators and helicases take on regulatory functions. Nat Rev Mol Cell Biol 2006; 7: 739-50.

23. Klein HL. The consequences of Rad51 overexpression for normal and tumor cells. DNA Repair (Amst) 2008; 7: 686-93.

24. Wiegmans AP, Al-Ejeh F, Chee N, et al. Rad51 supports triple negative breast cancer metastasis. Oncotarget 2014; 5: 3261-72

25. Di Sante G, Di Rocco A, Pupo C, Casimiro MC, Pestell RG. Hormone-induced DNA damage response and repair mediated by cyclin D1 in breast and prostate cancer. Oncotarget 2017; 8: 81803-12.

26. Eskandari E, Rezaifar A, Hashemi M. XPG Asp1104His, XRCC2 Rs3218536 A/G and RAD51 135G/C gene polymorphisms and colorectal cancer risk: a meta-analysis. Asian Pac J Cancer Prev 2017; 18: 1805-13.

27. Xie C, Drenberg C, Edwards H, et al. Panobinostat enhances cytarabine and daunorubicin sensitivities in AML cells through suppressing the expression of BRCA1, CHK1, and Rad51. PLoS One 2013; 8: e79106.

28. Ayremlou N, Mozdarani H, Mowla SJ, Delavari A. Increased levels of serum and tissue miR-107 in human gastric cancer: correlation with tumor hypoxia. Cancer Biomark 2015; 15: 851-60.

29. Takahashi Y, Forrest AR, Maeno E, Hashimoto T, Daub CO, Yasuda J. MiR-107 and MiR-185 can induce cell cycle arrest in human non small cell lung cancer cell lines. PLoS One 2009; 4: e6677.

30. Lee KH, Lotterman C, Karikari C, et al. Epigenetic silencing of MicroRNA miR-107 regulates cyclin-dependent kinase 6 expression in pancreatic cancer. Pancreatology 2009; 9: 293-301.

31. Wang S, Ma G, Zhu H, et al. miR-107 regulates tumor progression by targeting NF1 in gastric cancer. Sci Rep 2016; 6: 36531.

32. Gao B, Hao S, Tian W, et al. MicroRNA-107 is downregulated and having tumor suppressive effect in breast cancer by negatively regulating brain-derived neurotrophic factor. J Gene Med 2017;19. doi: 10.1002/jgm.2932.

33. Wang Y, Chen F, Zhao M, et al. MiR-107 suppresses proliferation of hepatoma cells through targeting HMGA2 mRNA 3'UTR. Biochem Biophys Res Commun 2016; 480: 455-60.

34. Wang P, Liu X, Shao Y, et al. MicroRNA-107-5p suppresses non-small cell lung cancer by directly targeting oncogene epidermal growth factor receptor. Oncotarget 2017; 8: 57012-23.

35. Huang JW, Wang Y, Dhillon KK, et al. Systematic screen identifies miRNAs that target RAD51 and RAD51D to enhance chemosensitivity. Mol Cancer Res 2013; 11: 1564-73.

36. Bhise NS, Chauhan L, Shin M, et al. MicroRNA-mRNA pairs associated with outcome in AML: from in vitro cellbased studies to AML patients. Front Pharmacol 2015; 6: 324.

37. Nikolic I, Andjelkovic M, Zaric M, et al. Induction of mitochondrial apoptotic pathway by raloxifene and estrogen in human endometrial stromal ThESC cell line. Arch Med Sci 2017; 13: 293-301. 